Kong. Res. J. 1(1) : 117-121, 2014

Kongunadu Arts and Science College, Coimbatore

\title{
PHYTOCHEMICAL STUDIES ON THE TERPENOIDS OF MEDICINALLY IMPORTANT PLANT SOLANUM VILLOSUM (MILL.) USING HPTLC
}

\author{
Venkatesh, R*., R. Vidya, B. Subbaiyan ${ }^{1}$, P. Samydurai ${ }^{1}$ and K. Kalaivani \\ Department of Biochemistry, ${ }^{1}$ Department of Botany, Kongunadu Arts and Science College (Autonomous), Coimbatore. \\ *Email: biovenki76@gmail.com.
}

\begin{abstract}
To determine terpenoid profile of Solanum villosum using high performance thin layer chromatography (HPTLC) technique. 2cl of test solution and $2 \mathrm{cl}$ of standard solution was loaded as $6 \mathrm{~mm}$ band length in the $3 \times 10$ Silica gel 60F254 TLC plate using Hamilton syringe and CAMAG LINOMAT 5 instrument. The extract was run along with the standard terpenoid compound and it was observed that the extract showed the presence of terpenoid and it was confirmed from the chromatogram after derivatization. The Rf value of the different compounds present in the extract was found to $0.04,0.21,0.27,0.55,0.59,0.65,0.75,0.80,0.90$ and 0.94 of peak 1, 2, 3, 4, 5, 6, 7, 8, 9 and 10 respectively. Among them, peaks 2, 6, 7 and 10 were found to be terpenoid compounds. It can be concluded that HPTLC analysis of ethanolic leaf extract of Solanum villosum (Mill) can be used as a diagnostic tool for the correct identification of the plant and it is useful as a phytochemical marker and also a good estimator of genetic variability in plant populations.
\end{abstract}

Key words: HPTLC, ethanol extract, Solanum villosum, phytochemicals, terpenoid.

\section{INTRODUCTION}

Solanum villosum (Mill.) belongs to family Solanaceae, it is commonly known as red-fruit nightshade, is widely distributed in many parts of India. Solanum is one of the most important and largest genera of the family Solanaceae comprising of about 84 genera and 3000 species were identified throughout the worldwide. The plant is an ayurvedic herb with multiple medicinal properties (Nandita Chowdhury et al., 2008).

The plant Solanum villosum contain many primary and secondary metabolites such as, alkaloids, flavonoids, phenols, saponins, tannins, terpenoids, steroids, carbohydrates, glycosides, amino acids and proteins. The genus of Solanum species contains excellent antioxidant properties and free radical scavenging ability (Annie Jacob and Radha, 2013).

The plants of $S$. nigrum complex has been traditionally used as an analgesic, antispasmodic, antiseptic, antidysentric, antinarcotic, emollient, diuretic, tonic, soporific, laxative, anticancer, antiulcer and for disorders of neuro-vegetative system etc. (Edmonds and Chweya, 1997). This medicinal value is mainly attributed to the alkaloid content of the plants.

The plant compounds mainly used for treating worms, cold, hoarseness of voice, fever, dysuria, enlargement of the liver, muscular pain, spleen and stone in the urinary bladder (Akilesh Sharma et al., 2011; Watt and Breyer-Brandwijk 1962; Aslanov and Novruzov 1978).
In the present study, HPTLC analysis of ethanolic leaf extract of Solanum villosum specifically for terpenoid profile was compared with the standards. The Solanum villosum and related species are widely used as leafy herbs and vegetables, as a source of fruit and for various medicinal purposes. In spite of known uses in traditional medicines, no documented evidence is available on terpenoid compound analysis. So the HPTLC analysis is to provide information for terpenoid content of the plant extract.

\section{MATERIALS AND METHODS}

\subsection{Plant material}

The leaves of the Solanum villosum (Mill.) plant were collected from Thadagam hills at Coimbatore district, Tamilnadu, India. The specimen sample was authenticated by Dr.V.S.Ramachandran, Associate Professor, Department of Botany, Bharathiar University, Coimbatore, Tamilnadu, India. The voucher specimen was deposited in the herbarium center, Department of Botany, Bharathiar University, Coimbatore.

\subsection{Extraction of plant material}

Plant materials thoroughly washed and shade dried at room temperature after that grind into powder was packed with No.1 Whatman filter paper and placed in soxhlet apparatus along with ethanol. The crude extract were collected and dried at room temperature, $30^{\circ} \mathrm{C}$ after which yield was weighed and then performed. 
2.3. HPTLC analysis of ethanolic extract of Solanum villosum (Mill)

Test solution preparation: The given ethanol extract $100 \mathrm{mg}$ was weighed in an electronic balance (Afcoset) and dissolved in $1 \mathrm{ml}$ ethanol and centrifuged at $3000 \mathrm{rpm}$ for $5 \mathrm{~min}$. This solution was used as test solution for HPTLC analysis.

\subsection{Sample application}

$2 \mathrm{cl}$ of test solution and $2 \mathrm{cl}$ of standard solution was loaded as $6 \mathrm{~mm}$ band length in the $3 \mathrm{x}$ 10 Silica gel 60F254 TLC plate using Hamilton syringe and CAMAG LINOMAT 5 instrument.

\subsection{Spot development}

The samples loaded plate was kept in TLC twin trough developing chamber (after saturated with Solvent vapor) with respective mobile phase (Terpenoid) and the plate was developed in the respective mobile phase up to $90 \mathrm{~mm}$.

\subsection{Photo-documentation}

The developed plate was dried by hot air to evaporate solvents from the plate. The plate was kept in Photo-documentation chamber (CAMAG REPROSTAR 3) and captured the images at White light, UV $254 \mathrm{~nm}$ and UV366 $\mathrm{nm}$.

\subsection{Derivatization}

The developed plate was sprayed with respective spray reagent (Terpenoid) and dried at $100^{\circ} \mathrm{C}$ in Hot air oven. The plate was photodocumented in Day light and UV 366nm mode using Photo-documentation (CAMAG REPROSTAR 3) chamber. Scanning Before derivatization, the plate was fixed in scanner stage (CAMAG TLC SCANNER 3) and scanning was done at UV $254 \mathrm{~nm}$. The Peak table, Peak display and Peak densitogram were noted. The software used was winCATS 1.3.4 version.

\subsection{Analysis details for Terpenoid}

Mobile phase: n-Hexane - Ethyl acetate (7.2 : 2.9). Spray reagent: Anisaldehyde sulphuric acid reagent.

\section{RESULTS AND DISCUSSION}

\subsection{HPTLC analysis of Terpenoids}

\subsubsection{Detection}

Blue, bluish violet coloured zones at Visible light mode present in the given standard and sample track observed in the chromatogram after derivatization, which confirmed the Presence of Terpenoid in the given standard and maybe in sample.
The ethanolic leaf extract of Solanum villosum was run along with the standard terpenoid compound and it was observed that the extract showed the presence of terpenoid and it was confirmed from the chromatogram after derivatization. The $\mathrm{Rf}$ value of the different compounds present in the extract was found to 0.04 , $0.21,0.27,0.55,0.59,0.65,0.75,0.80,0.90$ and 0.94 of peak $1,2,3,4,5,6,7,8,9$ and 10 respectively. Among them, peaks 2, 6, 7 and 10 were found to be terpenoid compounds.

The WHO has emphasized the need to ensure the quality of medicinal plant products by using modern controlled techniques and applying suitable standards (WHO, 1998). Modern chromatographic techniques like HPLC and HPTLC were used to judge the authenticity of traditional recommendations (Khan et al., 2009). The HPTLC method can be used for phytochemical profiling of plants and quantification of compounds present in plants, with increasing demand for herbal products as medicines and cosmetics, there is an urgent need for standardization of plant products (Pawar et al., 2010).

HPTLC finger print analysis has become the most potent tool for quality control of herbal medicines because of its simplicity and reliability. It can serve as a tool for identification, authentication and quality control of herbal drug (Mauji et al., 2011). HPTLC finger printing profile is useful as phytochemical marker and also a good estimation of genetic variability in plant populations. Thus the HPTLC fingerprint profiles of the major chemical constituents in the crude extract along with their Rf values and percentage proportions were recorded which would serve as a reference standard for the scientist who engaged in research on the medicinal properties of this plant (Johnson et al., 2011).

The phytochemical evaluation is one of the tools for the quality assessment, which includes preliminary phytochemical screening, chemo profiling and marker compound analysis using modern analytical techniques. In the last two decades, HPTLC has emerged as an important tool for the qualitative, semiqualitative and quantitative phytochemical analysis of herbal drugs and formulations. The major advantage of HPTLC is that several samples can be analysed simultaneously using a small quantity of mobile phase (Modi et al., 2008). HPTLC fingerprint profiles of the Solanum villosum leaf extract for terpenoids have been developed. Rf values and the relative percentage of the separated compounds were recorded. 
Table 1. HPTLC analysis of ethanolic leaf extract of Solanum villosum for Terpenoid profile

\begin{tabular}{llllll}
\hline Track & Peak & Rf & Height & Area & Assigned substance \\
\hline STD & 1 & 0.85 & 90.8 & 3196.5 & Terpenoid standard \\
Sample A & 1 & 0.04 & 541.7 & 13312.1 & Unknown \\
Sample A & 2 & 0.21 & 27.1 & 601.7 & Terpenoid 1 \\
Sample A & 3 & 0.27 & 85.7 & 2998.0 & Unknown \\
Sample A & 4 & 0.55 & 11.4 & 112.9 & Unknown \\
Sample A & 5 & 0.59 & 31.1 & 1464.8 & Unknown \\
Sample A & 6 & 0.65 & 15.3 & 270.2 & Terpenoid 2 \\
Sample A & 7 & 0.75 & 66.0 & 1939.4 & Terpenoid 3 \\
Sample A & 8 & 0.80 & 65.9 & 2166.5 & Unknown \\
Sample A & 9 & 0.90 & 26.9 & 868.9 & Unknown \\
Sample A & 10 & 0.94 & 21.4 & 459.8 & Terpenoid 4 \\
\hline
\end{tabular}

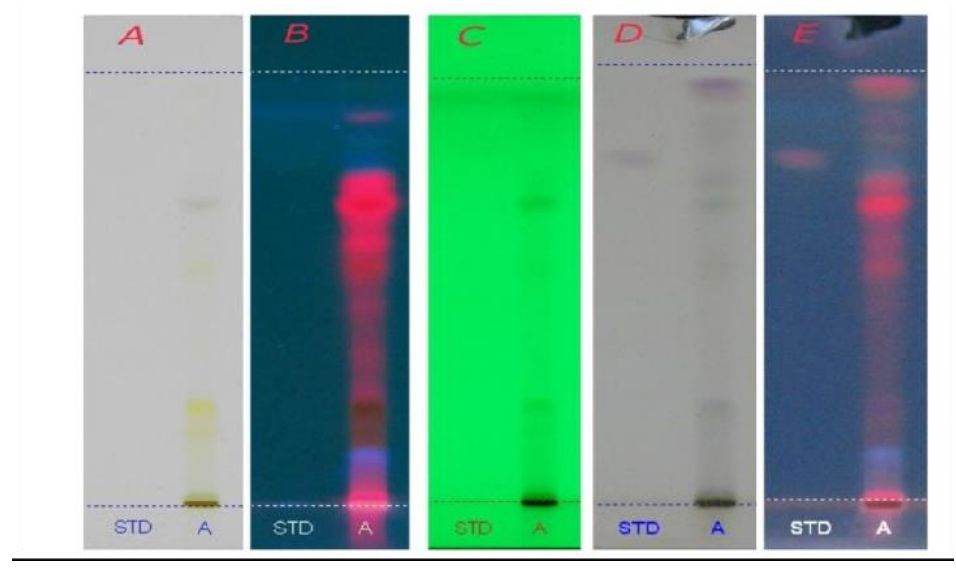

Figure 1. HPTLC studies on the terpenoid of medicinally important plant $S$. villosum

A: HPTLC of the ethanolic leaf extract of S. villosum under daylight. B: HPTLC of the ethanolic leaf extract of S. villosum under UV $366 \mathrm{~nm}$ C: HPTLC of the ethanolic leaf extract of S. villosum under UV $254 \mathrm{~nm}$. D: HPTLC of the ethanolic leaf extract of S. villosum under daylight - after derivatization. E: HPTLC of the ethanolic leaf extract of $S$. villosum under UV $366 \mathrm{~nm}$ after derivatization.

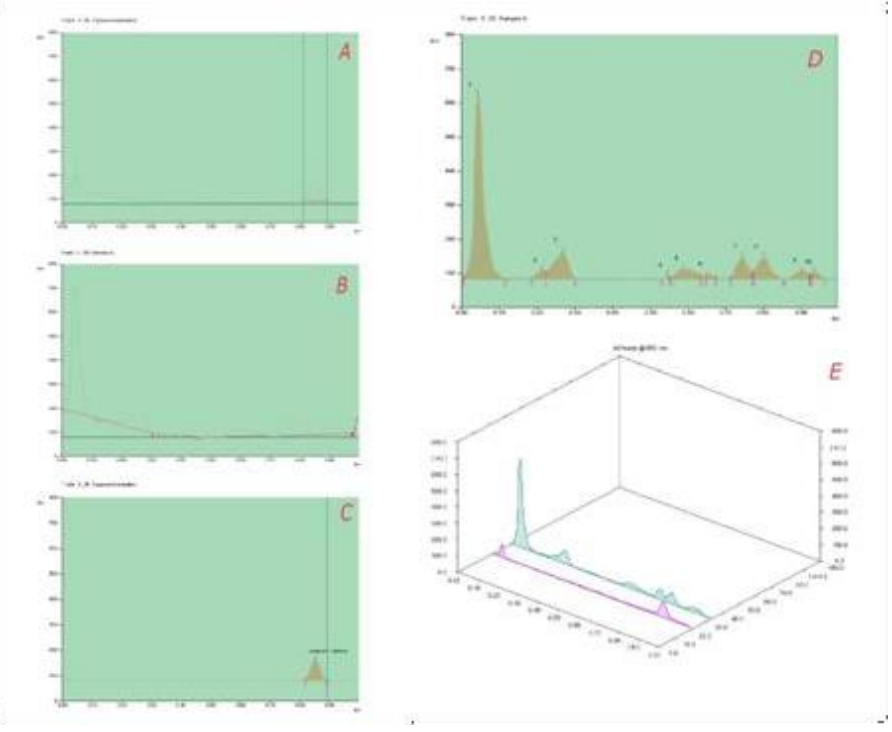

Figure 2. HPTLC chromatogram of ethanolic leaf extract of $S$. villosum (Mill.)

A: HPTLC chromatogram of Track STD - Terpenoid standard Baseline display (Scanned at 366nm), B: HPTLC chromatogarm of Track STD - Terpenoid standard Peak densitogram display (Scanned at 366nm), C: HPTLC chromatogarm of Track A - Sample A ethanolic leaf extract of S.villosum - Baseline display (Scanned at 366nm), D: HPTLC chromatogram of Track A - Sample A ethanolic leaf extract $S$. villosum - Peak densitogram display (Scanned at $500 \mathrm{~nm}$ ), E: HPTLC chromatogram of 3D display of all Tracks. 
Terpenoids composed of "isoprenoid" units constitute one of the largest group of natural products accounting for more than 40000 individual compounds, with several new compounds being discovered every year (Sacchettini and Poulter, 1997; Peñuelas and Munné-Bosch, 2005; Withers and Keasling, 2007). Most of the terpenoids are of plant origin; however, they are also synthesized by other organisms, such as bacteria and yeast as part of primary or secondary metabolism. Terpenoids are synthesized from two five-carbon building blocks, i.e., the isoprenoid units. Based on the number of building blocks, terpenoids are classified into several

classes, such as monoterpenes (e.g., carvone, geraniol, $d$-limonene, and perillyl alcohol), diterpenes (e.g. retinol and trans-retinoic acid), triterpenes e.g., betulinic acid (BA), lupeol, oleanic acid, and ursolic acid (UA), and tetraterpenes e.g., $\alpha$ carotene, $\beta$-carotene, lutein, and lycopene (Rabi and Bishayee, 2009).

The diverse array of terpenoid structures and functions has provoked increased interest in their commercial use. Terpenoids have been found to be useful in the prevention and therapy of several diseases, including cancer, and also to have antimicrobial, antifungal, antiparasitic, antiviral, anti-allergenic, antispasmodic, antihyperglycemic, antiinflammatory, and immunomodulatory properties (Wagner and Elmadfa, 2003; Sultana and Ata, 2008; Shah et al., 2008). In addition, terpenoids can be used as protective substances in storing agriculture products as they are known to have insecticidal properties (Theis and Lerdau, 2003).

\section{CONCLUSION}

It can be concluded that HPTLC analysis of ethanolic leaf extract of Solanum villosum (Mill.) for terpenoid profile can be used as a diagnostic tool for the correct identification of the plant and it is useful as a phytochemical marker and also a good estimator of genetic variability in plant populations.

\section{ACKNOWLEDGEMENTS}

The authors are thankful to our Secretary and Joint Secretary of Kongunadu Arts and Science College, Coimbatore, Tamilnadu, India for providing facilities and encouragement.

\section{REFERENCES}

Akilesh sharma, Mayank Swaroop sharma, Anurag Mishra, Janjay sharma, Brijesh kumar and Bhandari, (2011). A review on other plants used in liver diseases. IJRPC 1(2).
Annie Jacob and P. Radha, (2013). Effect of Solanum nigrum leaf extract on the enzymic Antioxidant status of tumor bearing mice. Int. J. of Research in Engineering and Bioscience 1: 9 - 23.

Aslanov, S.M. and E.N. Novruzov, (1978) Studies on glycoalkaloids of Solanum nigrum growing in Azerbaijan. Izvestiya. Akademil Nauk Azerbaidzhanskoi SSR, Biologicheskikh Nauk 3:15-18.

Edmonds, J.M. and J.A. Chweya, (1997). Black nightshades, Solanum nigrum (and related species). p. 113. 1. B.P.G.R. Rome and I.P.K Gatersleben.

Johnson, M., Y. Mariswamy and W.E. Gnaraj, (2011). Chromatographic fingerprint analysis of steroids in Aerva lanata L. by HPTLC technique. Asian Pac. J. of Trop. Biomed., 428-433.

Khan, M.S., N. Nema, M.D. Kharya and S. Khanam, (2009). Chromatographic estimation of maturity based phytochemical profiling of Ipomoea mauritiana, 1:22-30.

Mauji, R., M.Z. Abdin, M.A. Khan, and J. Prabhakar, (2011) . HPTLC fingerprint analysis: A Quality control of Authentication of Herbal Phytochemicals. Verlag Berlin Heidelberg: Springer, p.105.

Modi, K.P., N.M. Patel and R.K. Goyal, (2008). Estimation of L-topaf from Mucuma pruriens Linn. And formulation containing M pruriens by HPTLC method. Chem. Pharma. Bull., 56(3):357.

Nandita Chowdhury, Anupam Ghosh and Goutam Chandra, (2008). Mosquito larvicidal activities of Solanum villosum berry extract against the dengue vector Stegomyia aegypti. BMC Complementary and Alternative Medicine 8:10.

Pawar, R.K., S. Shivani, K.C. Singh and K.V. Sharma Rajeev, (2010). Physico-chemical standardization and development HPTLC method for the determination of Andrographonin in Kalmgh Navyas Loha. An Ayurvedic formulation. Bangladesh J. Pharmacol., 2(1): 295-301.

Peñuelas, J and S Munné-Bosch, (2005). Isoprenoids: an evolutionary pool for photoprotection. Trends Plant Sci. 10: 166-169.

Rabi,T and A. Bishayee, (2009). Terpenoids and breast cancer chemoprevention. Breast Cancer Res Treat. 115: 223-239.

Sacchettini, J.C. and C.D. Poulter, (1997). Creating isoprenoid diversity. Science; 277: 1788-1789. 
Shah, B.A., G.N. Qazi and S.C. Taneja, (2009). Boswellic acids: a group of medicinally important compounds. Nat Prod Rep 26: 72-89.

Sultana, N. and A. Ata, (2008). Oleanolic acid and related derivatives as medicinally important compounds. J Enzyme Inhib Med Chem 23: 739756.

Theis N. and M. Lerdau (2003). The evolution of function in plant secondary metabolites. Int $J$ Plant Sci 164: S93-S103.

Wagner, K.H. and I. Elmadfa, (2003). Biological relevance of terpenoids. Overview focusing on mono-, di- and tetraterpenes. Ann Nutr Metab 47: 95-106.

Watt, J.M. and M.G. Breyer-Brandwijk, (1962). Solanum nigrum L. p. 996-1000 in the Medicinal and Poisonous Plants of Southern and Eastern Africa. E. and S. Livingstone Ltd., Edinburgh and London.

WHO, (1998). Quality control method for medicinal plant material Geneva: WHO p.1-15.

Withers, S.T. and J.D. Keasling, (2007). Biosynthesis and engineering of isoprenoid small molecules. Appl Microbiol Biotechnol 73: 980-990. 